\title{
Performance Analysis of Underwater Swarm Sensor Networks
}

\author{
Marco Tabacchiera ${ }^{\S}$, Samuela Persia*£, Chiara Lodovisi ${ }^{\S}$, and Silvello Betti ${ }^{\S}$ \\ §University of Rome "Tor Vergata," v.le Politecnico 1, 00133 Rome, Italy. \\ *Fondazione Ugo Bordoni, v. B. Castiglione 59, 00142 Rome, Italy. \\ Email: betti.ing@uniroma2.it, spersia@fub.it, chiara.lodovisi@libero.it, marco.tabacchiera@uniroma2.it
}

\begin{abstract}
Fundamental key aspects of underwater acoustic communications have been taken into account to define an underwater swarm network for monitoring and exploration applications. We discuss the communication architecture of underwater sensor networks as well as the factors that influence underwater network design. Specifically, the main requirements needed to design a suitable sensors underwater system has been provided by considering a Cross-Layer solution among the lower layers of the node to maintain the power consumption as low as possible without complexity addition in the architecture design. Results show that the average power can be preserved thanks to power control implementation used to optimize the overall across the physical, Medium Access Control (MAC) and Network (NWK) layers. This study wants to provide the performance analysis of a swarm network model to provide guideline for hardware developing of swarm nodes for a real underwater application.
\end{abstract}

\section{INTRODUCTION}

In the last decades a growing interest has been showed for the Wireless Sensor Networks (WSNs) thanks to their versatility in a lot of different field applications. Among of all underwater communications have attracted in the last decade research and military community thank to the possibility to monitor selected ocean areas not reachable with wired systems. In addition, the advances in acoustic modem technology have led to a proliferation of underwater applications focused on communication between various remote devices within a network environment. Generally, a WSN is constrained by power consumption [1], whereas underwater systems encour additional constraints that require a carefully design of the final system.

In this work we consider a preliminary model of a sensor node for an underwater swarm network. This network is characterized by some difficulties, in terms of energy saving and bandwidth availability, that have to be taken into account during the definition phase. These limitations will influence both network design, and system architecture explanation. The ultimate objective of this work is then to highlight the fundamental basis for the development of new advanced communication techniques for efficient underwater communication and networking for enhanced monitoring and exploration applications. In particular, we considered the main aspects for the lower layers design of a possible swarm node model. We describe in details the solutions that we have taken into account and we evaluate them by performance analysis comparisons. Specifically, starting from an overview of the underwater acoustic system architecture and the associated physical, data link, and network layer issues, we try to provide which are challenges at each level to define the particular application under test: the swarm network.

In this context, particular attention has been showed in the last decade in the MAC solutions needed for underwater networks. New solutions have been provided in literature, where updated versions of the consolidate MAC have been proposed. For instance, MACA and FAMA schemes [4] are obtained to resolve drawbacks of the well known CSMA MAC. Actually, the efficiency of the former is impacted heavily by propagation delays, due to their multi-way handshakes that waste energy, and the last one has not been considered for nodes with mobility capability. Improved solutions have been proposed by considering MACA with WAIT command, or Slotted FAMA [7]. In addition, solutions in which power constraints are taken into account could be considered to preserve the lifetime of the network, such as S-MAC. Actually, it optimizes the minimal consumption of the network but does not consider bandwidth utilization and access delay as objectives. Furthermore this technique may not be suitable for an environment where dense sensor deployment cannot be assumed and with mobile nodes. Therefore these solutions are not suitable for network with mobility capability. Indeed performance analysis of this solutions have been provided in literature, but they consider shallow water scenario in which nodes are assumed fixed with anchors placed on the sea bottom [6]. The network that we intend investigate is different: it requires mobility of the nodes in a swarm manner and thus we can assume a high level of the connectivity in the monitored area (e.g., the maximum distance between two adjacent nodes for a typical swarm can be assumed as no more than $d=25 \mathrm{~m}$ ). By these consideration, and considering no very high traffic load, simple MAC schemes can be assumed, such as ALOHA or CSMA due to their capability to obtain good throughput level in a underwater network with reliable connection among the nodes as verified in [8]. In addition we implement power control without complexity addition in the MAC scheme in order to preserve the energy, by considering a Cross-layer approach through which the transmitted power is set at the minimum level to reach next node. This solution is possible thanks to the 

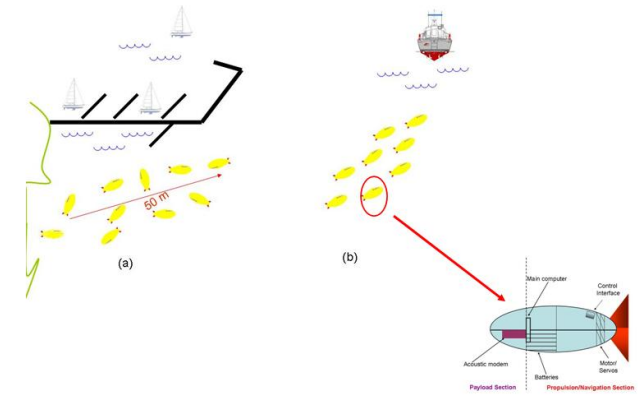

Fig. 1. Swarm mode operations: environment monitoring scenario (a), alarm detection scenario(b). On the right Scheme of a Swarm Node.

implementation of a geographic algorithm as routing scheme at the network layer: the Greedy Perimeter Stateless Routing algorithm [9]. The geographic techniques are very promising, even if it is still not clear how accurate localization information can be obtained in the underwater environment with limited energy expenditure. Anyway forwarding information by considering position information knowledge permits to preserve the power of the whole network by reducing useless redundant hops towards nodes far from the final destination.

The outcome of our study will be the basic concepts of the hardware design of the swarm nodes, which is the scope of our research in the next year.

The paper is organized as follow: a brief introduction of underwater swarm networks is provided in the second section, the application scenario which we have considered for our performance evaluations is described in the third section, the system model is provided in the fourth section, and the main results are showed in the fifth section. Finally, in the sixth section the main conclusions are drawn.

\section{UNDERWATER SENSOR NETWORK: AN OVERVIEW}

Underwater sensor networks have different factors influencing performance requirements with respect to terrestrial ones. Therefore, key challenges in underwater communications are needed and give motivations for different choice in the architecture protocol stack design. Generally, the challenges in the design of underwater acoustic networks are due to:

- Available bandwidth that is severely limited;

- Underwater channel that is severely impaired, especially due to multi-path and fading;

- Propagation delay that in underwater is five orders of magnitude higher than in radio frequency (RF) terrestrial channels, and extremely variable;

- High bit error rates and the temporary losses of connectivity (shadow zones) can be experienced, due to the extreme characteristics of the underwater channel;

- Battery power is limited and usually batteries cannot be recharged, also because solar energy cannot be exploited;
- Underwater sensors which are prone to failures because of fouling and corrosion.

All these factors determine the temporal and spatial variability of the acoustic channel, and make the available bandwidth of the Underwater Acoustic (UW-A) channel limited and dramatically dependent on both range and frequency [2]. Longrange systems that operate over several tens of kilometers may have a bandwidth of only a few $\mathrm{kHz}$, while a shortrange system operating over tens of meters may have up to a $\mathrm{MHz}$ bandwidth. Moreover, the communication range is dramatically reduced as compared to the terrestrial radio channel, and thus underwater communications lead to consider constraints never considered for the terrestrial networks into protocol choice at each layer to partially overcome limitations due to underwater environment.

\section{UNDERWATER SWARM DEFINITION}

WSNs are applications driven. It means that is not possible to consider a unique solution for all underwater applications. In this work we are interested in the definition of a particular underwater system: the underwater swarm network. This particular application is characterized by a network of nodes with mobility capability very close one to each other. A possible scheme of a node of the swarm is depicted in the fig.1. In the figure is possible to see the major functional components of the node. Generally, the node can be considered as consisting of two main components: a forward payload section, and a propulsion/control/navigation section. This network will extend in a very limited area, the maximum distance between the first and the last nodes of the swarm will be no more than $50 \mathrm{~m}$ and it will be characterized of high data rate up to 100 $\mathrm{kbit} / \mathrm{s}$ for very short distance. In addition, the structure of the network will be a distributed network in which nodes, through the exchange of control information, will take decisions in a collaborative manner.

This system will be able to work in two different modes:

- Environment Monitoring: in this situation nodes perform measurements of proper parameters, measurements of shallow water in the port area and short range communications are considered (see (a) part of fig.1).

- Alarm detection: the swarm detects an alarm situation, for instance a measured value of a specific parameter (e.g., oil in the water) is greater then the fixed threshold in a specific region, and thus it will be ready to coordinate itself and move towards the area in which the anomalies have been detected (see (b) part of fig.1).

For our performance studies, the considered solutions at each level of the system model, as explained in the following section, have been referred to the "swarm in alarm detection operation mode",.

\section{System MOdeL}

We consider a underwater network of 10 swarm nodes located in random positions within an area of $200 \mathrm{~m} \times 200 \mathrm{~m}$. Each node has mobility capability. All nodes within the communication range $r$ of a node are considered as its neighbors. 
Every node may generate traffic and forward packets to the more complex node, the sink node or Base Station (BS) in a multi-hop manner. The communication range $\mathrm{R}$ of the $\mathrm{BS}$ is supposed to cover all nodes, so that the BS can address each node directly, i.e. we assume that downlink communications are direct and without errors. The BS queries to each node of the network an information (e.g., temperature, oil, salinity measurements).

\section{A. Protocol Stack}

The node is modeled by considering the main suggestions proposed in literature. Our scope is to provide performance analysis of the proposed solutions in order to delineate a guide line for the actual realization of the swarm. In particular, we aim to have:

- low power consumption, i.e. a high average residual charge in the network after a significant time of operation with a uniform charge distribution over the whole network at PHY layer;

- low interference and collisions, i.e. low number of transmitted Data Frame and ACK packets to reduce both interference and power consumption to preserve lifetime of the network at MAC layer;

- low latency delay, i.e. low End to End Delay (EED) obtained by averaging the propagation time for each fragmented packet from source to destination at NWK and APPL layers, for future complex data to transmit (e.g. multimedia, video/imange data).

\section{B. PHY Layer}

As already mentioned, we consider a system based on sound propagation in the water. PHY choices strictly depend on the application scenarios. For this preliminary study we investigated the definition of "Transmission and data communication" swarm scenario. For data transmission no selective propagation is required and an isotropic antenna could be more efficient at the frequency carrier of $300 \mathrm{kHz}$. Low frequency means low attenuation but at the same time implies a more harmful multipath effect. More deeply we consider a system having an operational band $W=60 \mathrm{kHz}$ at a center frequency $f_{c}=300 \mathrm{kHz}$, a bit rate of $R_{b}=20 \mathrm{kbps}$ and a header length of $L_{h} \cong 48$ bits. An MFSK modulation is implemented, with $\mathrm{M}=4$.

The signal-to-noise ratio (SNR) associated with the received packets is calculated as:

$$
S N R=\frac{P_{T X} / A(T x, R x)}{N+N_{M U I}}
$$

where $P_{T X}$ is the transmitted power, $A(T x, R x)$ is the attenuation over the link between the transmitting node $T x$ and the receiving node $R x . N$ is the noise, and $N_{M U I}$ is the multiuser interference. The Gaussian approximation for $N_{M U I}$ is assumed. The attenuation is given as

$$
A(x)=x^{k} \cdot a^{x}
$$

where is the energy spreading factor ( $k$ is 1 for cylindrical, 1.5 for practical, and 2 for spherical spreading), and:

$$
a=10^{\frac{\alpha(f)}{10}}
$$

is a frequency-dependent term obtained from the absorption coefficient $\alpha(f)$. The absorption coefficient for the frequency range of interest is calculated according to Thorp's expression [3] expressed in $[d B / k m]$ and $f$ in $[k H z]$

$\alpha(f)=0.11 \frac{f^{2}}{1+f^{2}}+44 \frac{f^{2}}{4100+f^{2}}+2.75 \cdot 10^{-4} f^{2}+0.003$

A packet is correctly received if the SNR computed in (1) exceeds a proper threshold, which can be evaluated by considering the sensitivity level of the receiver. This value is obtained by considering data sheets specifications of the transducers considered in our model. Specifically, for our simulations two transducers have been considered: the Reson TC4034 and the ITC-1089D. The specifications of them are: Receiving Voltage Sensitivity (RVS) of $-218 \mathrm{~dB}$ (re $1 \mathrm{~V} / 1 \mu \mathrm{Pa}$ ), and Transmit Voltage Response (TVR) exceeding $122 \mathrm{~dB}$ (re $1 \mu \mathrm{Pa} / 1 \mathrm{~V} @$ $1 \mathrm{~m})$ for the former, and $\mathrm{RVS}=-216 \mathrm{~dB}($ re $1 \mathrm{~V} / 1 \mu \mathrm{Pa})$, TVR $=148 \mathrm{~dB}$ (re $1 \mu \mathrm{Pa} / 1 \mathrm{~V} @ 1 \mathrm{~m})$ for the latter, respectively. In order to establish the sensitivity level of the devices starting from these characteristic parameters, we used the following equation:

$$
R V S=20 \cdot \log (O C V)-I L
$$

where OCV (Open Circuit Voltage) is the output voltage from the transducer, while IL (Intensity Level) represents the acoustic intensity received. To understand what is the minimum output voltage received, we need to quantify the noise level and to calculate $\mathrm{OCV}$ in that condition. More deeply we considered the worst operation mode condition to calculate the noise, such as the presence of strong winds, high waves and naval activity. Turbulence and thermal noise, is also taken into account in this evaluation, and thus we estimated a noise at $-93 \mathrm{~dB}$ re $\mu P a$. From formula (5) we obtained the minimum value of $\mathrm{OCV}$, needed to estimate the sensitivity of the receiver. Holding fast to that value of OCV, we can establish the decision threshold for the correct received packets.

\section{MAC Layer}

Different solutions have been proposed in literature. The no complex solutions requirements have been suggested of the implementation of simple MAC scheme such as ALOHA or improved version of them. In particular, we compared the performance of two different MAC scheme in order to define which are the guideline for the access underwater medium scheme. We repeated the performance measurements by considering: the ALOHA and the CSMA MAC. We investigated how the solution adopted can influence the performance of the system. 


\section{NWK Layer}

The routing algorithm used is a typical geographic algorithm, the GPSR. This type of protocols establishes sourcedestination paths by leveraging localization information, i.e., each node selects its next hop based on the position of its neighbors and of the destination node. These techniques requires two strict constraints: the algorithm is more sensitive to node density [10]; the nodes need of localization information with respect to an absolute reference, such as a GPS navigation system. In addition node's position is current affected and thus an error could be taken into account when the packets are forwarded. In this preliminary step a 2D dimension the position we have considered. In the next future routing schemes that account for the 3D underwater environment need to be developed. Especially, in the 3D case the effect of currents should become evident, since the intensity and the direction of currents are dependent on the depth of the sensor node. Thus, underwater currents can modify the relative position of sensor devices and also cause connectivity holes, especially when ocean column monitoring is performed in deep waters. However, the geographic choice may be preferable twofold aspects: reduce the redundant multi-hops because by selecting only next hop closest the destination and thus reduce the whole power consumptions the network; reduce further the power consumption by setting the transmitting power at the minimum level necessary to reach the next hop. The latter consideration assumes a cross-layer paradigm between the MAC and the NWK allows the MAC to share the knowledge of the next selected hop, which is chosen by the routing algorithm within the NWK. With this scheme, the actual transmitted power is generally lower than the maximum allowable power, leading to a preservation of nodes battery charge.

\section{E. Mobility}

An important aspect that should be considered in our analysis is the mobility of nodes. The model to which we refer at this stage of analysis is the Random Way Point (RWP) [11]. The RWP model is one of the most widely used mobility models in performance analysis of mobile wireless networks. It is an elementary model which describes the movement pattern of independent nodes by simple terms. This model is used in terrestrial networks, where the rate is fixed, then the position remains constant and does not change. For successive instants of time, punctuated by a parameter called "updateinterval", the position of the node is reproposed. In water, even if the speed is set, the position varies due to sea currents. In this first phase we put ourselves in a perfect condition (as in terrestrial networks). In the next future, to simulate the move by a swarm, we will refer to Manhattan Propagation Model [12].

\section{TEST CASES}

Traditionally WSNs for data transmission depends on power availability of each node for two purposes: to permit the forwarding data in multi-hop manner between source and destination of the information, and the connectivity of the network. In this situation to preserve the lifetime of each node is the main constraint of the network. For underwater transmission the latency represents an important constraint as the power consumption. It is due to the nature of the propagation medium. This means that some solutions suitable for terrestrial transmission becomes unacceptable for underwater transmission. Therefore, performance analysis has been performed in order to consider which are factors that deeply influence the sensor network reliability. We remind that our study is a preliminary study to hardware design of a swarm sensor network suitable for underwater applications. For this reason we investigate the behavior of a preliminary model in order to define solutions more adapt for this emerging sensor network.

More deeply, we define the following test cases, as representative of exemplary scenarios of increasing complexity:

- ALOHA MAC - We consider a CBR traffic with an ALOHA scheme is assumed at MAC layer.

- CSMA MAC - We repeat the performance evaluation by considering a Carrier Sense MAC scheme.

We repeated the simulations by consider different velocity of the node of the swarm, and the different traffic load of the network. Furthermore, we consider the effect of the interference of the other nodes of the network during the activity to consider the performance evaluation in a real context, because the presence of other nodes permits to improve on only the connectivity of the network but, at the same time increases the interference. This issue might be carefully taken into account for a real-time traffic because it requires high delivery over longer times with high QoS. By simulations we want to define suitable trade-offs among different factors at each layer of the node.

\section{PERFormance ANALYSiS AND TRADE-OFFS}

Dynamic system simulations have been carried out in Omnet++ and system performance compared for the different test cases described in the previous section. We implemented the above described system in the Mobility Framework of Omnet++. For details about the simulation implementation we refer the interested reader to [14].

We used it as starting point for our analysis. Specifically we used the model of the node to create our Swarm node. The model of both the simple node and the network under test is depicted in Fig.1. Simulations have been performed for different speed mobility of the node and for different traffic load. For each case under study, we run a set of five stochastic discrete-event simulations, each having a duration of ten thousand seconds. The performance figures are then averaged over the five snapshots. Averaging over simulation snapshots, obtained with different nodes' positions in the area and thus different connection links, allows us to obtain a significant confidence interval for our statistical analysis [15].

Performance have been measured in terms of:

- residual charge - the average residual charge of all nodes in the network at the end of the simulation run; 
- Delivery success rate - the percentage of data packets correctly received by the destination node (the BS) at the end of the simulation run;

We assume that the position information are stored in all nodes, and thus a perfect knowledge of exact neighbors' positions is available at any time without control traffic exchange among neighbor nodes.

\section{A. Residual Charge}

The residual charge of nodes is evaluated in order to quantify the impact of the different type of the traffic over the power consumption. The power consumption of each node depends on its own activities. The initial charge, at the beginning of the simulation, of each node is assumed as $10 \mathrm{mAh}$. Specifically, we firstly define analytical models to take into account charge discharge, and after we compared them with results obtained by simulations.

- Analytical evaluation- To transmit a data packet from source to destination we can consider to different operation mode: Direct Access strategy, and Multi-hop strategy respectively. We can evaluate the power consumption for both solutions by taking into account the following considerations: the power level needed to transmit a data packet from one node to another over a distance $r / N$, is $P_{1}=P_{0} \cdot A(r / N)$ for the entire packet duration $T_{p}$. Hence, the total consumed energy for transmission over $N$ hops is according to analytical evaluations performed in [4]:

$$
E_{\text {hop }}=P_{0} A\left(\frac{r}{N}\right) T_{p} \frac{N(N+1)}{2}
$$

for the Multi-hop strategy and:

$$
E_{d i r}=P_{0} T_{p} \sum_{i=1}^{N} A\left(\frac{i r}{N}\right)
$$

for the Direct Access strategy respectively. $P_{0}$ is the minimum received power to obtain a desired quality of reception, $A$ is the attenuation according to equation (2), and $r$ the coverage range of each node.

A comparison of both strategies for the swarm is depicted in the Fig.2 in order to appreciate the effect of the relay strategy. We considered no more than $50 \mathrm{~m}$ as the maximum realistic distance that two nodes among the swarm could reach. Different network sizes have been considered in this analysis from $N=1$, which is the simplest case, where the network is composed only by two elements: the master collecting data node, and the underwater sensor; to $N=10$ where the network is composed by 10 sensor performing measurements nodes, and a collecting node. By analysis, we observed that for direct access the energy required to transmit data toward destination is independent by the number of the node in the network, and thus the trend of the energy level increases with the distance regardless the increase of the simple nodes in the network. On the other hand, by relay strategy it is possible to appreciate the

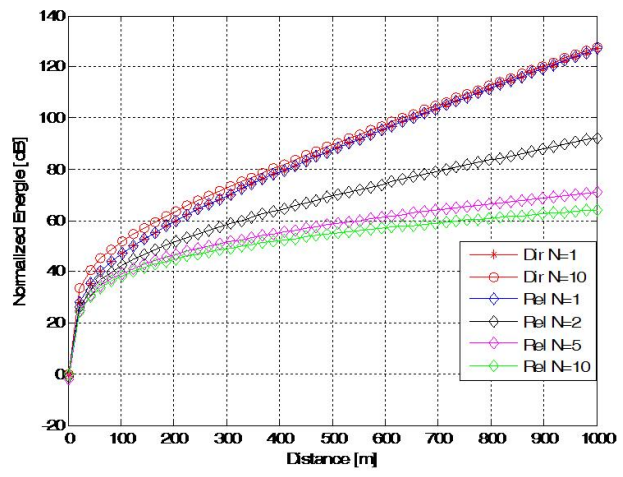

Fig. 2. comparison of direct and relay strategy in terms of total normalized (to $P_{0} T_{p}$ ) energy needed to transmit a packet from the more distant sensor node to the master node for an underwater swarm network. The parameter on the curves is network size in terms of number of simple nodes considered in the network, and consequently the number of hops needed.

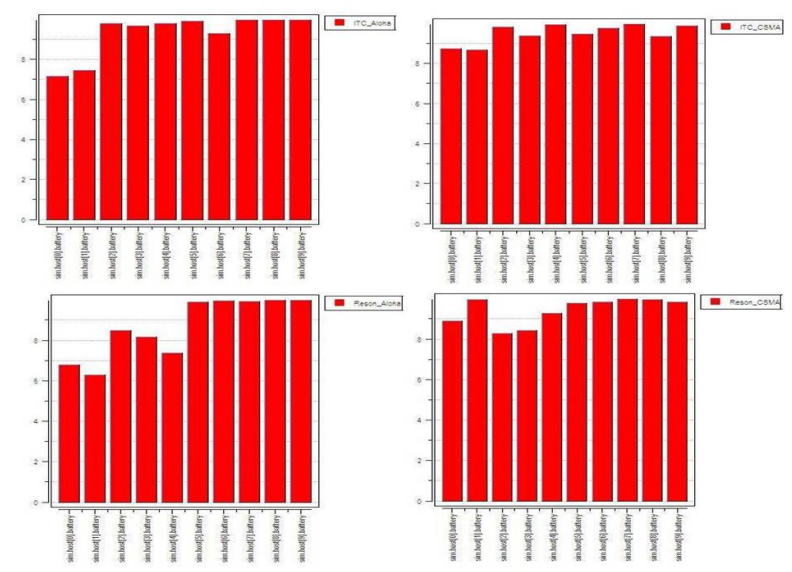

Fig. 3. comparison of two MAC schemes and devices of the residual charge at the end of ten simulation runs. For all simulations mobility of nodes of $1.5 \mathrm{~m} / \mathrm{s}$, and low traffic load have been assumed.

enhancement introduced by the increase of the number of hops. Specifically, for $\mathrm{N}=10$, by using relay is possible to spend half level of the energy needed with respect to the energy needed for the direct access. The latter has the same energy consumption regardless the number of simple nodes present in the network, because this strategy does not take advantage by the presence of other nodes and thus spends energy to cover alone the whole distance.

- Simulations evaluation-In the Fig.3 the residual charge for exemplary test cases under study, by using multi-hop strategy, is evaluated. By simulations we verified that the power control implementation is able to obtain a more uniform discharge of the nodes in CSMA case than the ALOHA ones. In any cases the Reson device experiments a slight more power consumption than the ICT device. 

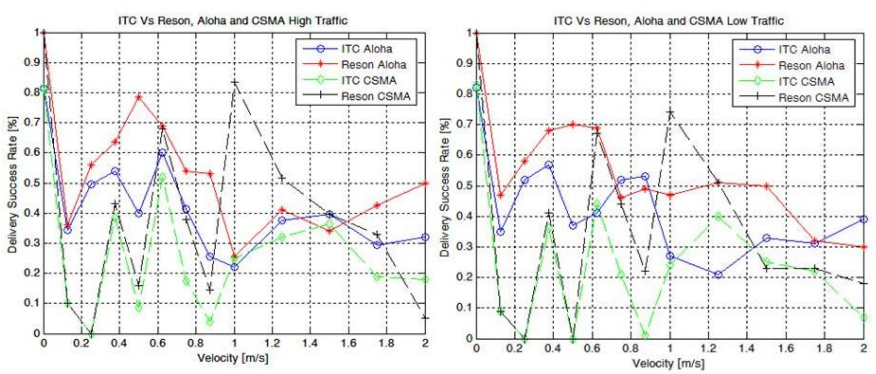

Fig. 4. Delivery Rate vs speed of nodes for different devices and different MAC schemes. Two different traffic loads have been considered.

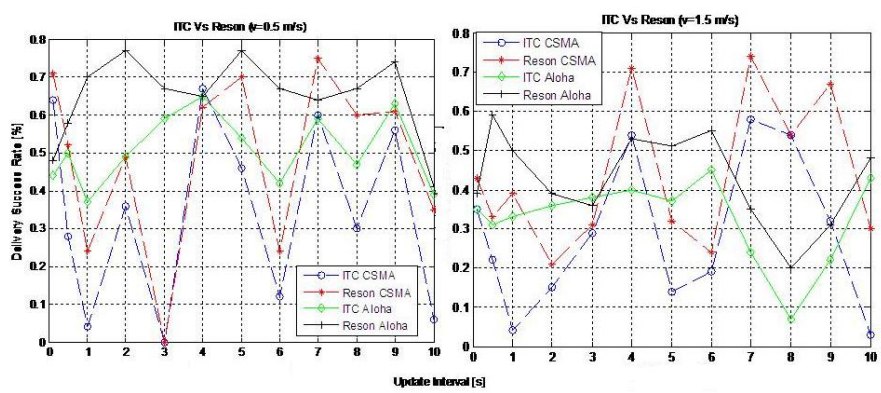

Fig. 5. Delivery Rate vs updating information of the speed among nodes by using different device and MAC schemes. The speed of nodes assumed are $0.5 \mathrm{~m} / \mathrm{s}$ (on the left side) and $1.5 \mathrm{~m} / \mathrm{s}$ (on the right side).

\section{B. Delivery Success Rate}

The impact of the MAC considered for underwater applications is evaluated in terms of the delivery success rate for the different test cases described previously, as a function of both mobility of the nodes and traffic load (see Fig.4). By simulations we noted that the use of ALOHA leads to performance improvements regardless the traffic load with respect to the CSMA solution. This trend confirmed results found in literature by considering different underwater scenario such as "shallow water" scenario [13]. Anyway for both schemes the Reson devices seems to obtain better results than the ITC1089D.

After we evaluated, for two speeds of nodes, the delivery rate vs. the updating information of the nodes position. We remind that we consider, at this step, an ideal situation in which no fluctuant effect due to the sea is considered, and thus we assumed that the position information obtained by each node is reliable. This study wants to be a benchmark in order to define updating rate requirements to take into account for next design phase of the swarm nodes. Fig. 5 shows that a trade-off between the updating information and speed of nodes permits to obtain good performance by using the Reson TC4034 device. In particular, we can say that, for low mobility, the ALOHA scheme is preferable than the CSMA ones. The opposite trend is verified when speed increases. These considerations confirm that the scheme strictly depends on the application scenario under test.

\section{CONCLUSION}

Underwater sensor networks have been considered in this work, in order to define design requirements to be satisfied to obtain an efficient underwater system. Special attention has been shown for data link layer. Performance evaluations by comparing different speeds and traffic loads for a swarm network have been performed. By extensive system simulations we verified that the performance strictly depends on the solutions considered at the different protocol layer. Specifically, we compared two different MAC layer, and transducers. In the next future we consider a more detailed system in which the mobility of the node will be taken into account in more realistic way. In particular, a 3 dimensions motion will be considered with a Manhattan model to define the mobility of the swarm in alert situation.

\section{ACKNOWLEDGMENT}

This work supported by "HARNESS" (Human telecontrolled Adaptive Robotic Network of SensorS) National Project.

\section{REFERENCES}

[1] I.F. Akyildiz at all, "A survey on sensor networks," Communications Magazine, IEEE 2002, Vol. 40, Issue 8, pp. 102 - 114.

[2] I. F. Akyildiz, D. Pompili, T. Melodia, "Challenges for Efficient Communication in Underwater Acoustic Sensor Networks," ACM Sigbed Review, vol. 1, no. 2, July 2004

[3] L. Berkhovskikh and Y. Lysanov, "Fundamentals of Ocean Acoustics," New York: Springer, 1982.

[4] E.M. Sozer, M. Stojanovic, J.G. Proakis, "Underwater acoustic networks," Oceanic Engineering, IEEE Journal of, 2000, vol. 25, pp. 72-83.

[5] J.G. Proakis, et. al. "Shallow Water Acoustic Networks," IEEE Communications Magazine, November 2001, pp. 114-119

[6] C. Petrioli, R. Petroccia, M. Stojanovic, "A comparative performance evaluation of MAC protocols for underwater sensor networks," Proc. OCEANS 2008, pp. 1-10.

[7] M. Molins and M. Stojanovic, "Slotted FAMA: A MAC Protocol for Underwater Acoustic Networks," Submitted to Proc. IEEE OCEANS'06 Conf., 2006.

[8] S. M. Smith and J. C. Park, "A peer-to-peer communication protocol for underwater acoustic communication," in OCEANS'97, Oct. 1997, pp. 268-272.

[9] B. Karp, H.T. Kung, "GPSR: Greedy Perimeter Stateless Routing for Wireless Netwrorks," Proc. of the 6th Annual Intern. Conf. on Mobile Comput. and Network., pp. 243-254, Aug., 2000, Boston, MA, USA.

[10] F. Kuhn, R. Wattenhofer, Y. Zhang, and A. Zollinger, "Geometric adhoc routing: of theory and practice," Proc. of the 22nd Annual Symp. on Principles of Distributed Computing PODC '03. ACM Press, New York, NY, pp.63-72. Boston, MA, July, 2003.

[11] J. Broch et.al., "A Performance Comparison of Multi-Hop Wireless Ad Hoc Network Routing Protocols," In Proceedings of the Fourth Annual ACM/IEEE International Conference on Mobile Computing and Networking (MobiCom 98), Dallas, Texas, Aug.1998.

[12] J. E. Berg, " A Recursive Method For Street MicroCell Path Loss Calculation," PIMRC 1995, Sep.1995,Toronto,Canada.

[13] B. Peleato, M. Stojanovic, "A MAC protocol for ad-hoc Underwater Acoustic Sensor Networks," Proc. WUWNet 2006, pp. 113-115.

[14] www.omnetpp.org/

[15] K. Pawlikowski, H.-D.J. Jeong, J.-S.R. Lee, "On credibility of simulation studies of telecommunication networks", IEEE Commun. Mag., vol. 40, no. 1, pp. 132-139, Jan. 2002. 